\title{
Defensive prediction with expert advice
}

\author{
Vladimir Vovk \\ vovk@cs.rhul.ac.uk \\ http://vovk.net
}

March 9, 2019

\begin{abstract}
The theory of prediction with expert advice usually deals with finite or finite-dimensional pools of experts. In this paper we give similar results for pools of decision rules belonging to an infinite-dimensional functional space which we call the Fermi-Sobolev space. For example, it is shown that for a wide class of loss functions (including the standard square, absolute, and log loss functions) the average loss of the master algorithm, over the first $N$ steps, does not exceed the average loss of the best decision rule with a bounded Fermi-Sobolev norm plus $O\left(N^{-1 / 2}\right)$. Our proof techniques are very different from the standard ones and are based on recent results about defensive forecasting. Given the probabilities produced by a defensive forecasting algorithm, which are known to be well calibrated and to have high resolution in the long run, we use the Expected Loss Minimization principle to find a suitable decision.
\end{abstract}

\section{Introduction}

This paper further develops a new approach to probability forecasting which we call "defensive forecasting". This approach has several appealing features. First, for every constructive law of probability expressed in game-theoretic terms there exists a procedure of defensive forecasting that satisfies this law perfectly 13. In particular, one can construct a prediction algorithm with high degrees of calibration and resolution in the long term, which is mathematically expressed by explicit inequalities without making any assumptions on the data-generating mechanism [11]. This paper describes a third feature: defensive forecasts lead to efficient decisions; in particular, defensive forecasting allows one to obtain new results in prediction with expert advice.

First papers on prediction with expert advice with general loss functions (e.g., [1, 8]) dealt with finite pools of experts. The next step was to consider finite-dimensional pools of experts (e.g., 3, 6, 9]). This paper continues with infinite-dimensional pools of experts. To get an idea of its central results, the reader is advised to start from Corollaries [3 


\section{The N91 algorithm}

In this paper we will use a modification of the defensive forecasting algorithm introduced in [11] and called N91. We will not give an explicit description of the algorithm (which can be found in 11]) and only describe one of its properties.

Our prediction protocol is:

FOR $n=1,2, \ldots$ :

Reality announces $x_{n} \in[0,1]^{K}$.

Forecaster announces $p_{n} \in[0,1]$.

Reality announces $y_{n} \in\{0,1\}$.

END FOR.

At each step $n$ Forecaster observes the $K$ attributes of an object $x_{n}$ and is asked to predict its label $y_{n}$. N91 is Forecaster's strategy in this protocol (or prediction algorithm). In Theorem 1 below we will state N91's property, but before that we will need to give several auxiliary definitions. We will be following [11].

A function $f:[0,1]^{k} \rightarrow \mathbb{R}$ is Lipschitzian if

$$
\sup _{u, v \in[0,1]^{k}, u \neq v} \frac{|f(u)-f(v)|}{|u-v|}<\infty .
$$

For such a function the cross derivative

$$
g\left(t_{1}, \ldots, t_{k}\right):=\frac{\partial^{k} f\left(t_{1}, \ldots, t_{k}\right)}{\partial t_{1} \cdots \partial t_{k}}
$$

is well defined almost everywhere. The $L_{2}$ norm $\left(\int g^{2}(t) \mathrm{d} t\right)^{1 / 2}$ of $g$ is denoted $S(f)$, and we define the Fermi-Sobolev norm $\|f\|_{\mathrm{FS}}$ by

$$
\|f\|_{\mathrm{FS}}^{2}:=\sum_{m=0}^{k} \sum_{\left\{i_{1}, \ldots, i_{m}\right\} \subseteq\{1, \ldots, k\}} S^{2}\left(\int_{0}^{1} \cdots \int_{0}^{1} f\left(t_{1}, \ldots, t_{k}\right) \mathrm{d} t_{i_{1}} \cdots \mathrm{d} t_{i_{m}}\right),
$$

where $\int_{0}^{1} \cdots \int_{0}^{1} f\left(t_{1}, \ldots, t_{k}\right) \mathrm{d} t_{i_{1}} \cdots \mathrm{d} t_{i_{m}}$ is considered as a function of the free variables. The reader might find it helpful to concentrate on the one-dimensional case, $k=1$, at the first reading; the Fermi-Sobolev squared norm then reduces to

$$
\|f\|_{\mathrm{FS}}^{2}:=\left(\int_{0}^{1} f(t) \mathrm{d} t\right)^{2}+\int_{0}^{1}\left(f^{\prime}(t)\right)^{2} \mathrm{~d} t
$$

the first addend reflecting how centered $f$ is and the second measuring the volatility of $f$.

Theorem 1 ([1] ) The predictions $p_{n}$ output by the N91 algorithm satisfy

$$
\left|\sum_{n=1}^{N}\left(y_{n}-p_{n}\right) f\left(p_{n}, x_{n}\right)\right| \leq\left(\frac{2}{\sqrt{3}}\right)^{K+1}\|f\|_{\mathrm{FS}} \sqrt{N}
$$

for all $N$ and all Lipschitzian functions $f:[0,1]^{K+1} \rightarrow \mathbb{R}$. 
The right-hand side of (1) is $O(\sqrt{N})$; this term will be as ubiquitous in this paper as it is in the traditional theory (see, e.g., [1] and 4]). In all our results the ratio $2 / \sqrt{3}$ can be replaced by its upper bound 1.155 .

Theorem 1 will be useful in our preliminary discussion in the next section, but much stronger results can be derived from its simple modifications, which will be stated in the appendix.

\section{Preliminary discussion}

In this section we will introduce our decision making protocol, give several definitions needed for the following sections, and prove a simple result (Proposition 11) showing how defensive forecasting is relevant to decision making. A much more useful result will be proved in the next section.

The decision making protocol of this paper is:

FOR $n=1,2, \ldots$ :

Reality announces $x_{n} \in[0,1]^{K}$.

Decision Maker announces $\gamma_{n} \in \Gamma$.

Reality announces $y_{n} \in\{0,1\}$.

END FOR.

Decision Maker chooses his actions from a nonempty decision space $\Gamma$ and his performance is measured using a loss function $\lambda:\{0,1\} \times \Gamma \rightarrow \mathbb{R}$; his average loss over the first $N$ steps is

$$
\frac{1}{N} \sum_{n=1}^{N} \lambda\left(y_{n}, \gamma_{n}\right) .
$$

In tradition of prediction with expert advice, Decision Maker will compete against a pool of decision rules, where a decision rule is defined to be a function $D:[0,1]^{K} \rightarrow \Gamma$; the average loss suffered by such a decision rule is

$$
\frac{1}{N} \sum_{n=1}^{N} \lambda\left(y_{n}, D\left(x_{n}\right)\right) .
$$

The exposure $\operatorname{Exp}_{D}:[0,1]^{K} \rightarrow \mathbb{R}$ of the decision rule $D$ at a point $x \in[0,1]^{K}$ is defined by

$$
\operatorname{Exp}_{D}(x):=\lambda(1, D(x))-\lambda(0, D(x)) .
$$

A choice function is any function mapping $[0,1]$ to $\Gamma$; intuitively, such a function maps a probability distribution $(1-p, p)$ on $\{0,1\}$ to a decision $\gamma=\gamma(p)$ that is optimal or nearly optimal under this probability distribution. The defect of a choice function $G$ is

$$
\Delta_{G}:=\sup _{p \in[0,1]}\left(\lambda(p, G(p))-\inf _{\gamma \in \Gamma} \lambda(p, \gamma)\right)
$$


where $\lambda(p, \gamma)$ is the expected loss caused by taking the decision $\gamma$ when the probability of 1 is $p$ :

$$
\lambda(p, \gamma):=p \lambda(1, \gamma)+(1-p) \lambda(0, \gamma) .
$$

If $\Gamma=[0,1]$ and the choice function $G(p):=p$ has zero defect, the loss function is called a proper scoring rule (e.g., the square loss and log loss functions, discussed in Section[5] are known to be proper scoring rules). The exposure $\operatorname{Exp}_{G}:[0,1] \rightarrow$ $\mathbb{R}$ of the choice function $G$ is defined by

$$
\operatorname{Exp}_{G}(p):=\lambda(1, G(p))-\lambda(0, G(p)) .
$$

We will be interested in decision rules and, in this section, choice functions whose exposure is a Lipschitzian function.

We will construct decision strategies from N91's probabilities by minimizing the expected loss. In some cases instead of the literal minimization we might want to use slightly suboptimal (but, e.g., continuous) choice functions. Therefore, we will use the following "soft" version of the Expected Loss Minimization principle: the decision strategy (i.e., Decision Maker's strategy) based on a choice function $G$ and a prediction algorithm is the one that outputs $\gamma_{n}:=G\left(p_{n}\right)$ at the $n$th step, where $p_{n}$ is the prediction output by the prediction algorithm.

Proposition 1 The decision strategy based on a choice function $G$ with Lipschitzian $\operatorname{Exp}_{G}$ and the N91 algorithm outputs decisions $\gamma_{n}$ such that

$$
\begin{aligned}
& \frac{1}{N} \sum_{n=1}^{N} \lambda\left(y_{n}, \gamma_{n}\right) \leq \frac{1}{N} \sum_{n=1}^{N} \lambda\left(y_{n},\right.\left.D\left(x_{n}\right)\right) \\
&+\left(\frac{2}{\sqrt{3}}\right)^{K+1} \frac{\left\|\operatorname{Exp}_{D}\right\|_{\mathrm{FS}}+\left\|\operatorname{Exp}_{G}\right\|_{\mathrm{FS}}}{\sqrt{N}}+\Delta_{G}
\end{aligned}
$$

for all $N$ and all decision rules $D$ with Lipschitzian $\operatorname{Exp}_{D}$.

Proof Notice that

$$
\lambda(y, \gamma)-\lambda(p, \gamma)=(y-p)(\lambda(1, \gamma)-\lambda(0, \gamma))
$$

always holds (this can be checked by subtracting (2) from $\lambda(y, \gamma):=y \lambda(1, \gamma)+$ $(1-y) \lambda(0, \gamma))$. In conjunction with Theorem 1 this implies

$$
\begin{aligned}
\sum_{n=1}^{N} \lambda\left(y_{n}, \gamma_{n}\right) & =\sum_{n=1}^{N} \lambda\left(y_{n}, G\left(p_{n}\right)\right) \\
= & \sum_{n=1}^{N} \lambda\left(p_{n}, G\left(p_{n}\right)\right)+\sum_{n=1}^{N}\left(\lambda\left(y_{n}, G\left(p_{n}\right)\right)-\lambda\left(p_{n}, G\left(p_{n}\right)\right)\right) \\
= & \sum_{n=1}^{N} \lambda\left(p_{n}, G\left(p_{n}\right)\right)+\sum_{n=1}^{N}\left(y_{n}-p_{n}\right)\left(\lambda\left(1, G\left(p_{n}\right)\right)-\lambda\left(0, G\left(p_{n}\right)\right)\right)
\end{aligned}
$$




$$
\begin{gathered}
\leq \sum_{n=1}^{N} \lambda\left(p_{n}, G\left(p_{n}\right)\right)+(2 / \sqrt{3})^{K+1}\left\|\operatorname{Exp}_{G}\right\|_{\mathrm{FS}} \sqrt{N} \\
\leq \sum_{n=1}^{N} \lambda\left(p_{n}, D\left(x_{n}\right)\right)+N \Delta_{G}+(2 / \sqrt{3})^{K+1}\left\|\operatorname{Exp}_{G}\right\|_{\mathrm{FS}} \sqrt{N} \\
=\sum_{n=1}^{N} \lambda\left(y_{n}, D\left(x_{n}\right)\right)-\sum_{n=1}^{N}\left(\lambda\left(y_{n}, D\left(x_{n}\right)\right)-\lambda\left(p_{n}, D\left(x_{n}\right)\right)\right) \\
+N \Delta_{G}+(2 / \sqrt{3})^{K+1}\left\|\operatorname{Exp}_{G}\right\|_{\mathrm{FS}} \sqrt{N} \\
=\sum_{n=1}^{N} \lambda\left(y_{n}, D\left(x_{n}\right)\right)-\sum_{n=1}^{N}\left(y_{n}-p_{n}\right)\left(\lambda\left(1, D\left(x_{n}\right)\right)-\lambda\left(0, D\left(x_{n}\right)\right)\right) \\
+N \Delta_{G}+(2 / \sqrt{3})^{K+1}\left\|\operatorname{Exp}_{G}\right\|_{\mathrm{FS}} \sqrt{N} \\
\leq \sum_{n=1}^{N} \lambda\left(y_{n}, D\left(x_{n}\right)\right)+(2 / \sqrt{3})^{K+1}\left\|\operatorname{Exp}_{D}\right\|_{\mathrm{FS}} \sqrt{N} \\
+N \Delta_{G}+(2 / \sqrt{3})^{K+1}\left\|\operatorname{Exp}_{G}\right\|_{\mathrm{FS}} \sqrt{N}
\end{gathered}
$$

which completes the proof.

The chain (3) demonstrates our proof technique very well; to show that the actual loss of our decision strategy does not exceed the actual loss of a decision rule $D$ by much, we notice that:

- the actual loss $\sum_{n=1}^{N} \lambda\left(y_{n}, G\left(p_{n}\right)\right)$ of our decision strategy is approximately equal, by Theorem 1] to the (one-step-ahead conditional) expected loss $\sum_{n=1}^{N} \lambda\left(p_{n}, G\left(p_{n}\right)\right)$ of our strategy;

- since we used the Empirical Loss Minimization principle, the expected loss of our strategy does not exceed the expected loss of $D\left(\right.$ assuming $\left.\Delta_{G}=0\right)$;

- the expected loss of $D$ is approximately equal to its actual loss (again by Theorem (1).

It will be convenient to introduce the following terminology (very imprecise, to be used in informal discussions only). We say that a prediction algorithm has good calibration-cum-resolution if the left-hand side of (1) is small for a wide class of functions $f:[0,1]^{K+1} \rightarrow \mathbb{R}$ and large $N$. We say that the algorithm has good calibration if

$$
\left|\frac{1}{N} \sum_{n=1}^{N}\left(y_{n}-p_{n}\right) f\left(p_{n}\right)\right| \ll 1
$$

for a wide class of functions $f:[0,1] \rightarrow \mathbb{R}$ and large $N$. Finally, we say that the algorithm has good resolution if

$$
\left|\frac{1}{N} \sum_{n=1}^{N}\left(y_{n}-p_{n}\right) f\left(x_{n}\right)\right| \ll 1
$$


for a wide class of $f:[0,1]^{K} \rightarrow \mathbb{R}$ and for large $N$.

\section{General result}

The main purpose of the previous section was to motivate the somewhat less natural definitions of this section. Our approach so far suffered from two drawbacks:

- The inequality in Proposition 1 can be tightened if we notice that in applying (11) to establishing the first and third inequalities in (3) we need not arbitrary Lipschitzian $f=f(p, x)$ but only $f=f(p)$ (known in advance) and $f=f(x)$. (In particular, we only need calibration and resolution separately, not calibration-cum-resolution.)

- The requirement that the exposure of the choice function should be continuous (even Lipschitzian) is very restrictive: for example, in the case of the absolute loss function (described in Subsection 5.2), there is no perfect (i.e., with $\Delta_{G}=0$ ) choice function $G$ with continuous exposure.

These drawbacks will be dealt with in Appendix A. To deal with the second one, we will have to modify the N91 algorithm so that it outputs extended predictions $\left(p_{n}, q_{n}\right) \in[0,1]^{2}$, where $p_{n}$ is the prediction of $y_{n}$, as before, and the extra component $q_{n}$ will make it possible to design perfect choice functions with continuous exposure.

The lexicographic square $£$ is defined to be the set $[0,1]^{2}$ equipped with the following linear order: if $\left(x_{1}, y_{1}\right)$ and $\left(x_{2}, y_{2}\right)$ are two points in $£,\left(x_{1}, y_{1}\right)<$ $\left(x_{2}, y_{2}\right)$ means that either $x_{1}<x_{2}$ or $x_{1}=x_{2}, y_{1}<y_{2}$. (Cf. [2], Problem 3.12.3(d).) The topology on the lexicographic square is, as usual, generated by the open intervals

$$
(a, b):=\{u \in £ \mid a<u<b\},
$$

$a$ and $b$ ranging over $£$. As a topological space, the lexicographic square is normal ([2], Problem 1.7.4(d)), compact ([2], Problem 3.12.3(a), [5], Problem 5.C), and connected ([2], Problem 6.3.2(a), [5], Problem 1.I(d)).

Now we redefine a choice function to be any function $G$ mapping the lexicographic square $£$ to $\Gamma$; in all our examples, it will map a probability distribution $(1-p, p)$ on $\{0,1\}$ and the extra "tie-breaking" component $q$ to an optimal decision, under this probability distribution. The defect of $G$ is

$$
\Delta_{G}:=\sup _{(p, q) \in £}\left(\lambda(p, G(p, q))-\inf _{\gamma \in \Gamma} \lambda(p, \gamma)\right),
$$

where $\lambda(p, \gamma)$ is defined by (2). In the next section, we will be interested in zero-defect choice functions. The exposure $\operatorname{Exp}_{G}: £ \rightarrow \mathbb{R}$ of $G$ is now defined as

$$
\operatorname{Exp}_{G}(p, q):=\lambda(1, G(p, q))-\lambda(0, G(p, q))
$$


The following theorem describes a property of the Empirical Loss Minimization principle applied to a modification of the N91 algorithm which outputs, at each step, a pair $\left(p_{n}, q_{n}\right) \in £$.

Theorem 2 The decision strategy based on a choice function $G$ with continuous $\operatorname{Exp}_{G}$ and a certain prediction algorithm outputting $\left(p_{n}, q_{n}\right)$ produces decisions $\gamma_{n}$ such that

$$
\begin{aligned}
& \sum_{n=1}^{N} \lambda\left(y_{n}, \gamma_{n}\right) \leq \sum_{n=1}^{N} \lambda\left(y_{n}, D\left(x_{n}\right)\right) \\
& +\left(\left(\frac{2}{\sqrt{3}}\right)^{K}\left\|\operatorname{Exp}_{D}\right\|_{\mathrm{FS}}+1\right) \sqrt{\sum_{n=1}^{N} p_{n}\left(1-p_{n}\right)\left(\operatorname{Exp}_{G}^{2}\left(p_{n}, q_{n}\right)+1\right)}+N \Delta_{G}
\end{aligned}
$$

for all $N$ and all decision rules $D$ with Lipschitzian $\operatorname{Exp}_{D}$.

This theorem will be proved in Appendix A.

\section{$5 \quad$ Examples}

By a game we will mean a pair $(\Gamma, \lambda)$, where $\Gamma$ is the decision space and $\lambda$ : $\{0,1\} \times \Gamma \rightarrow \mathbb{R}$ is the loss function. For all games considered in this section decisions $\gamma \in \Gamma$ may be interpreted as predictions, but we will still refer to them as decisions to avoid confusion with, e.g., N91's forecasts.

\subsection{The square loss game}

If $\Gamma=[0,1]$ and $\lambda(y, \gamma)=(y-\gamma)^{2}$, we have

$$
\lambda(1, D(x))-\lambda(0, D(x))=(1-D(x))^{2}-(D(x))^{2}=1-2 D(x)
$$

and, for $G(p, q):=p$,

$$
\lambda(1, G(p, q))-\lambda(0, G(p, q))=(1-p)^{2}-p^{2}=1-2 p .
$$

Therefore, $\left|\operatorname{Exp}_{G}\right| \leq 1$, and so Theorem 2 implies

Corollary 1 In the square loss game, there is a decision strategy that guarantees that, for all $N$ and all Lipschitzian decision rules $D$,

$$
\sum_{n=1}^{N} \lambda\left(y_{n}, \gamma_{n}\right) \leq \sum_{n=1}^{N} \lambda\left(y_{n}, D\left(x_{n}\right)\right)+\frac{1}{\sqrt{2}}\left(\frac{2^{K+1}}{3^{K / 2}}\left\|D^{\dagger}\right\|_{\mathrm{FS}}+1\right) \sqrt{N}
$$

where $D^{\dagger}:=D-1 / 2$ is the "centered" decision rule. 


\subsection{The absolute loss game}

For this game, $\lambda(y, \gamma)=|y-\gamma|$ with $\Gamma=[0,1]$. We find

$$
\lambda(p, \gamma)=p(1-\gamma)+(1-p) \gamma=p+(1-2 p) \gamma,
$$

and so the ideal decision would be

$$
\gamma:= \begin{cases}0 & \text { if } p<1 / 2 \\ 1 & \text { if } p>1 / 2\end{cases}
$$

with an arbitrary choice in the case of a tie, $p=1 / 2$. This is a discontinuous function of $p$, but we can instead use the following continuous choice function on the lexicographic square:

$$
G(p, q):= \begin{cases}0 & \text { if } p<1 / 2 \\ 1 & \text { if } p>1 / 2 \\ q & \text { if } p=1 / 2\end{cases}
$$

Since

$$
\lambda(1, \gamma)-\lambda(0, \gamma)=1-2 \gamma,
$$

$\operatorname{Exp}_{D}$ is the same as in the square loss case, (5), and

$$
\operatorname{Exp}_{G}(p, q)=1-2 G(p, q)= \begin{cases}1 & \text { if } p<1 / 2 \\ -1 & \text { if } p>1 / 2 \\ 1-2 q & \text { if } p=1 / 2\end{cases}
$$

Therefore, $\left|\operatorname{Exp}_{G}\right| \leq 1$, and we have the following corollary of Theorem 2

Corollary 2 In the absolute loss game, there is a decision strategy that produces decisions $\gamma_{n}$ such that, for all $N$ and all Lipschitzian D, (6) holds.

\subsection{The simple loss game}

In this subsection we mainly limit ourselves to informal discussions.

The loss function for the simple loss game is the same as for the absolute loss game, $\lambda(y, \gamma)=|y-\gamma|$, but $\Gamma=\{0,1\}$. Now the approach we have used so far does not work: since $\Gamma$ consists of two elements, there is no non-trivial continuous choice function $G: £ \rightarrow \Gamma$ (every continuous image of $£$ is connected: [2], Theorem 6.1.4).

A natural idea (1]) is to allow Decision Maker to use randomization. The expected loss of an algorithm making decision 1 with probability $\gamma$ and 0 with probability $1-\gamma$ is $|y-\gamma|$, where $y$ is the observed label; therefore, for the simple loss game a randomized decision strategy can guarantee the following analogue of (6):

$$
\sum_{n=1}^{N} \mathbb{E} \lambda\left(y_{n}, \gamma_{n}\right) \leq \sum_{n=1}^{N} \lambda\left(y_{n}, D\left(x_{n}\right)\right)+\frac{1}{\sqrt{2}}\left(\frac{2^{K+1}}{3^{K / 2}}\left\|D^{\dagger}\right\|_{\mathrm{FS}}+1\right) \sqrt{N}
$$


where $\mathbb{E}$ refers to the strategy's internal randomization (the decision rules $D$ can be allowed to take values in $[0,1])$.

The disadvantage of (8) is that typically we are interested in the strategy's actual rather than expected loss. Our derivation of (8) shows the role of randomization: with the choice function (7) no randomization is required unless $p=1 / 2$. Typically, we rarely find ourselves in a situation of complete uncertainty, $p_{n}=1 / 2$; therefore, only a little bit of randomization is needed, essentially for tie breaking. The actual loss will be very close to the expected loss. (It is instructive to compare this with the discussion of Dawid's example in 13, Subsection 4.4.)

\subsection{The log loss game}

For the log loss game, $\Gamma=[0,1]$ and

$$
\lambda(y, \gamma)= \begin{cases}-\ln \gamma & \text { if } y=1 \\ -\ln (1-\gamma) & \text { if } y=0\end{cases}
$$

(we temporarily, in this subsection only, allow $\lambda$ to take the value $\infty$; cf. the discussion at the end of this subsection). As in the square loss case, we use the "perfect" choice function $G(p, q):=p$. Since

$$
\lambda(1, \gamma)-\lambda(0, \gamma)=-\ln \gamma+\ln (1-\gamma)=\ln \frac{1-\gamma}{\gamma}
$$

and

$$
\begin{array}{r}
\sum_{n=1}^{N} p_{n}\left(1-p_{n}\right)\left(\left(\ln \frac{1-p_{n}}{p_{n}}\right)^{2}+1\right) \leq N \max _{p \in[0,1]} p(1-p)\left(\left(\ln \frac{1-p}{p}\right)^{2}+1\right) \\
\approx 0.526 N \leq 0.53 N
\end{array}
$$

we obtain from Theorem 2

Corollary 3 Some decision strategy in the log loss game produces decisions $\gamma_{n}$ such that, for all $N$ and all $D$ with Lipschitzian $\ln \frac{1-D}{D}$,

$$
\sum_{n=1}^{N} \lambda\left(y_{n}, \gamma_{n}\right) \leq \sum_{n=1}^{N} \lambda\left(y_{n}, D\left(x_{n}\right)\right)+0.73\left(\left(\frac{2}{\sqrt{3}}\right)^{K}\left\|\ln \frac{1-D}{D}\right\|_{\mathrm{FS}}+1\right) \sqrt{N}
$$

There is an obvious bug in our proof of Corollary 3 the log loss function takes values in $(-\infty, \infty]$ (since $\lambda(0,1)=\lambda(1,0)=\infty$ ), whereas Theorem [2 assumes that $\lambda$ takes values in $\mathbb{R}$. This will be corrected in Appendix B.

\subsection{Convex games}

One can generalize Corollaries 103 making them less precise (viz., involve an unspecified constant). In this subsection we consider games $(\Gamma, \lambda)$ such that $\Gamma$ 
is nonempty and

$$
C_{0}:=\inf _{\gamma \in \Gamma} \lambda(0, \gamma), \quad C_{1}:=\inf _{\gamma \in \Gamma} \lambda(1, \gamma)
$$

are finite. As usual, $\lambda$ is assumed to take values in $\mathbb{R}$; therefore, to cover the $\log$ loss game we redefine its decision space to $\Gamma:=(0,1)$. It is convenient (see, e.g., 4]) to summarize a game by its superdecision set

$$
\Sigma:=\left\{(x, y) \in \mathbb{R}^{2} \mid \exists \gamma \in \Gamma: x \geq \lambda(0, \gamma) \text { and } y \geq \lambda(1, \gamma)\right\}
$$

elements of this set will be called superdecisions. Superdecisions of the form $(\lambda(0, \gamma), \lambda(1, \gamma))$ will sometimes be called decisions. We will assume, additionally, that the set $\Sigma \subseteq \mathbb{R}^{2}$ is convex and closed. The Eastern tail of the game is the function

$$
\begin{aligned}
f:\left[C_{0}, \infty\right) & \rightarrow \mathbb{R} \cup\{\infty\} \\
x & \mapsto \inf \{y \mid(x, y) \in \Sigma\}-C_{1}
\end{aligned}
$$

and its Northern tail is

$$
\begin{aligned}
g:\left[C_{1}, \infty\right) & \rightarrow \mathbb{R} \cup\{\infty\} \\
y & \mapsto \inf \{x \mid(x, y) \in \Sigma\}-C_{0},
\end{aligned}
$$

where, as usual, inf $\emptyset:=\infty$; it is clear that $f$ and $g$ are nonnegative everywhere and finite on $\left(C_{0}, \infty\right)$ and $\left(C_{1}, \infty\right)$, respectively.

Corollary 4 Let the game $(\Gamma, \lambda)$ be such that $\Gamma \neq \emptyset$, (9) are finite, the superdecision set $\Sigma$ is convex and closed, and the tails $f$ and $g$ satisfy

$$
f_{+}^{\prime}(t)=O\left(t^{-2}\right), \quad g_{+}^{\prime}(t)=O\left(t^{-2}\right)
$$

as $t \rightarrow \infty$, where $f_{+}^{\prime}$ and $g_{+}^{\prime}$ stand for the right derivatives (see, e.g., [7], Section 23) of $f$ and $g$. There exist a constant $C$ and a decision strategy producing decisions $\gamma_{n}$ such that

$$
\sum_{n=1}^{N} \lambda\left(y_{n}, \gamma_{n}\right) \leq \sum_{n=1}^{N} \lambda\left(y_{n}, D\left(x_{n}\right)\right)+C\left(\left(\frac{2}{\sqrt{3}}\right)^{K}\left\|\operatorname{Exp}_{D}\right\|_{\mathrm{FS}}+1\right) \sqrt{N}
$$

always holds, for all $N$ and all $D$ with Lipschitzian $\operatorname{Exp}_{D}$.

This corollary is applicable to the square, absolute, and log loss games; for the log loss game, this follows from the fact that its tails satisfy

$$
f^{\prime}(t)=g^{\prime}(t)=-\frac{1}{e^{t}-1} \sim-e^{-t}=O\left(t^{-2}\right) .
$$

Remark If the loss function $\lambda$ is bounded, (10) holds trivially. The right derivatives in (10) can be replaced by left derivatives, since $\left|f_{+}^{\prime}\right| \leq\left|f_{-}^{\prime}\right|$ and $\left|g_{+}^{\prime}\right| \leq\left|g_{-}^{\prime}\right|$ (see, e.g., [7, Theorem 24.1). Condition (10) can be interpreted as saying that the tails should shrink fast enough. The case $f(t)=g(t)=t^{-1}$ can be considered borderline; Corollary 4 is still applicable in this case, but it ceases to be applicable for tails that shrink less fast. 


\section{$6 \quad$ Further research}

Two of the most natural directions in which this paper's results can be developed are:

- to extend Theorem 2 and its corollaries to the multi-class case (defensive forecasting for this case is discussed in [12]);

- to formally analyze games with non-convex loss functions along the lines of Subsection 5.3

\section{References}

[1] Nicolò Cesa-Bianchi, Yoav Freund, David Haussler, David P. Helmbold, Robert E. Schapire, and Manfred K. Warmuth. How to use expert advice. Journal of the Association for Computing Machinery, 44:427-485, 1997.

[2] Ryszard Engelking. General Topology, volume 6 of Sigma Series in Pure Mathematics. Heldermann, Berlin, second edition, 1989. First edition: 1977 (Państwowe Wydawnictwo Naukowe, Warsaw).

[3] Yoav Freund. Predicting a binary sequence almost as well as the optimal biased coin. In Proceedings of the Ninth Annual Conference on Computational Learning Theory, pages 89-98, New York, 1996. Association for Computing Machinery.

[4] Yuri Kalnishkan and Michael V. Vyugin. The Weak Aggregating Algorithm and weak mixability. In Proceedings of the Eighteenth Annual Conference on Learning Theory, 2005. To appear.

[5] John L. Kelley. General Topology. Van Nostrand, Princeton, NJ, 1957.

[6] Jyrki Kivinen and Manfred K. Warmuth. Exponential Gradient versus Gradient Descent for linear predictors. Information and Computation, 132:163, 1997.

[7] R. Tyrrell Rockafellar. Convex Analysis. Princeton University Press, Princeton, NJ, 1970.

[8] Vladimir Vovk. Aggregating strategies. In Mark Fulk and John Case, editors, Proceedings of the Third Annual Workshop on Computational Learning Theory, pages 371-383, San Mateo, CA, 1990. Morgan Kaufmann.

[9] Vladimir Vovk. Competitive on-line statistics. International Statistical Review, 69:213-248, 2001.

[10] Vladimir Vovk. Non-asymptotic calibration and resolution: smoothing approach, The Game-Theoretic Probability and Finance project, http://probabilityandfinance.com, Working Paper \#11, November 2004 . 
[11] Vladimir Vovk. Non-asymptotic calibration and resolution. Technical Report arXiv:cs.LG/0506004, arXiv.org e-Print archive, June 2005.

[12] Vladimir Vovk, Ilia Nouretdinov, Akimichi Takemura, and Glenn Shafer. Defensive forecasting for linear protocols. Technical Report arXiv:cs.LG/0506007, arXiv . org e-Print archive, June 2005.

[13] Vladimir Vovk, Akimichi Takemura, and Glenn Shafer. Defensive forecasting. Technical Report arXiv:cs.LG/0505083, arXiv.org e-Print archive, May 2005. Also published in the Proceedings of the Tenth International Workshop on Artificial Intelligence and Statistics, January 6-8, 2005, Savannah Hotel, Barbados.

\section{Appendix A: Proof of Theorem 2}

The main part of this paper is essentially self-contained; we tried to give all definitions and state all results clearly (although we did not prove quite a few of them). This appendix, however, assumes that the reader has read [1] and makes constant references to that paper.

\section{Nomenclature}

Before we start the proof we remind and make more systematic our terminology. The K29 algorithm was described in [1] (Section 2). The modified algorithm introduced in 11] (Appendix B) allowed us (11], Theorem 1) to improve the performance bound for K29 by bringing in factors $p_{n}\left(1-p_{n}\right)$; we will call the modified algorithm K29*.

A suitable parameter for this algorithm, called the N91 kernel, was also defined in 11 (Equation (6)). The K29 algorithm applied to the N91 kernel was called the N91 algorithm in [1]; analogously, we could call the K29* algorithm applied to the N91 kernel the $N 91^{*}$ algorithm. (These last two terms will not be used in the rest of this paper.) The N91 kernel is a kernel on $[0,1] \times \mathbf{X}$, where the object space $\mathbf{X}$ is $[0,1]^{K}$ and $K$ is the number of attributes. In the construction of the N91 kernel on $[0,1]^{K+1}$ the fact that the first coordinate of the points in $[0,1]^{K+1}$ was special (corresponding to the forecast rather than an attribute) was never used, and we could carry out the same construction taking only the attributes into account, with $K+1$ replaced by $K$. The resulting kernel will be called the object N91 kernel.

In the rest of this paper we will introduce several modifications of the K29* algorithm: $£ \mathrm{~K} 29^{*}, £_{\mathrm{K}} 29^{*}, £^{\dagger} \mathrm{K} 29^{*}$, and $£^{\dagger} \mathrm{K} 29^{*}$. In the proof of Theorem 2 we will use the $£ \mathrm{~K} 29^{*}$ algorithm applied to the combined feature mapping $\left(\Phi_{0}, \Phi_{1}\right)$, where $\Phi_{0}$ is the exposure of the choice function and $\Phi_{1}$ is the feature mapping leading to the object N91 kernel, but this will be explained in detail later in this appendix. 


\section{Preliminary results}

We start from an extension of our analysis of the K29 algorithm presented in [11. The following lemma will play the role of the Intermediate Value Theorem, used in [11.

Lemma 1 If a continuous function $f: £ \rightarrow \mathbb{R}$ takes both positive and negative values, there exists $x \in £$ such that $f(x)=0$.

Proof A continuous image of a connected compact set is connected (2], Theorem 6.1.4) and compact ([2], Theorem 3.1.10). Therefore, $f(£)$ is a closed interval.

A kernel $\mathbf{k}$ on $£ \times \mathbf{X}$ is admissible if the functions $\mathbf{k}\left((p, q, x),\left(p^{\prime}, q^{\prime}, x^{\prime}\right)\right)$ and $\mathbf{k}((p, q, x),(p, q, x))$ are continuous in $(p, q) \in £$. For such a kernel the function

$$
\begin{aligned}
S_{n}(p, q):=\sum_{i=1}^{n-1} \mathbf{k}\left(\left(p, q, x_{n}\right),\left(p_{i}, q_{i}, x_{i}\right)\right) & \left(y_{i}-p_{i}\right) \\
+ & \frac{1}{2} \mathbf{k}\left(\left(p, q, x_{n}\right),\left(p, q, x_{n}\right)\right)(1-2 p)
\end{aligned}
$$

is continuous in $(p, q) \in £$.

LeXicographic K29* ALGORIthm (£K29*)

Parameter: admissible kernel $\mathbf{k}$ on $£ \times \mathbf{X}$

FOR $n=1,2, \ldots$ :

Read $x_{n} \in \mathbf{X}$.

Define $S_{n}(p, q)$ by (11), $(p, q) \in £$.

Output any root $(p, q)$ of $S_{n}(p, q)=0$ as $\left(p_{n}, q_{n}\right)$;

if there are no roots,

set $p_{n}:=\left(1+\operatorname{sign} S_{n}\right) / 2$ and set $q_{n}$ to any number in $[0,1]$.

Read $y_{n} \in\{0,1\}$.

END FOR.

(Notice that sign $S_{n}$ is well defined by Lemma 1) By Mercer's theorem there exists a function $\Phi: £ \times \mathbf{X} \rightarrow H$ (a feature mapping taking values in an inner product space $H$ ) such that

$$
\mathbf{k}(a, b)=\Phi(a) \cdot \Phi(b), \quad \forall a, b \in £ \times \mathbf{X} .
$$

We will say that $\Phi$ is admissible if the kernel $\mathbf{k}$ defined by (12) is admissible. Now we can prove the following version of Theorem 1 in [11].

Theorem 3 Let $\mathbf{k}$ be the kernel defined by [12) for an admissible feature mapping $\Phi: £ \times \mathbf{X} \rightarrow H$. The lexicographic K29* algorithm with parameter $\mathbf{k}$ outputs $\left(p_{n}, q_{n}\right)$ such that

$$
\left\|\sum_{n=1}^{N}\left(y_{n}-p_{n}\right) \Phi\left(p_{n}, q_{n}, x_{n}\right)\right\|^{2} \leq \sum_{n=1}^{N} p_{n}\left(1-p_{n}\right)\left\|\Phi\left(p_{n}, q_{n}, x_{n}\right)\right\|^{2}
$$


always holds for all $N=1,2, \ldots$.

Proof We will follow the proof of Theorem 1 in [1] (Appendix A).

The only change in Binary Forecasting Game I is that Forecaster's move becomes $\left(p_{n}, q_{n}\right) \in £$ (in particular, the extra component $q_{n}$ does not enter the formula for the increase in Skeptic's capital; its only role is to help Skeptic behave in a continuous way). The only changes in Binary Forecasting Game II are that Skeptic's move becomes a continuous function $S_{n}: £ \rightarrow \mathbb{R}$ and Forecaster's move becomes $\left(p_{n}, q_{n}\right) \in £$; accordingly, $S_{n}\left(p_{n}\right)$ is replaced by $S_{n}\left(p_{n}, q_{n}\right)$ in the formula for updating $\mathcal{K}_{n}$. Because of Lemma 1 Lemma 1 of 11 still holds.

Following the $£ \mathrm{~K} 29^{*}$ algorithm Forecaster ensures that Skeptic will never increase his capital with the strategy

$$
\begin{aligned}
s_{n}:=\sum_{i=1}^{n-1} \mathbf{k}\left(\left(p_{n}, q_{n}, x_{n}\right),\left(p_{i},\right.\right. & \left.\left.q_{i}, x_{i}\right)\right)\left(y_{i}-p_{i}\right) \\
& +\frac{1}{2} \mathbf{k}\left(\left(p_{n}, q_{n}, x_{n}\right),\left(p_{n}, q_{n}, x_{n}\right)\right)\left(1-2 p_{n}\right) .
\end{aligned}
$$

Using the formula

$$
\left(y_{n}-p_{n}\right)^{2}=p_{n}\left(1-p_{n}\right)+\left(1-2 p_{n}\right)\left(y_{n}-p_{n}\right)
$$

(which can be checked by setting $y_{n}:=0$ and $y_{n}:=1$ ), we can see that the increase in Skeptic's capital when he follows (14) is

$$
\begin{aligned}
\mathcal{K}_{N}-\mathcal{K}_{0}= & \sum_{n=1}^{N} s_{n}\left(y_{n}-p_{n}\right) \\
= & \sum_{n=1}^{N} \sum_{i=1}^{n-1} \mathbf{k}\left(\left(p_{n}, q_{n}, x_{n}\right),\left(p_{i}, q_{i}, x_{i}\right)\right)\left(y_{n}-p_{n}\right)\left(y_{i}-p_{i}\right) \\
& +\frac{1}{2} \sum_{n=1}^{N} \mathbf{k}\left(\left(p_{n}, q_{n}, x_{n}\right),\left(p_{n}, q_{n}, x_{n}\right)\right)\left(1-2 p_{n}\right)\left(y_{n}-p_{n}\right) \\
= & \frac{1}{2} \sum_{n=1}^{N} \sum_{i=1}^{N} \mathbf{k}\left(\left(p_{n}, q_{n}, x_{n}\right),\left(p_{i}, q_{i}, x_{i}\right)\right)\left(y_{n}-p_{n}\right)\left(y_{i}-p_{i}\right) \\
& -\frac{1}{2} \sum_{n=1}^{N} \mathbf{k}\left(\left(p_{n}, q_{n}, x_{n}\right),\left(p_{n}, q_{n}, x_{n}\right)\right)\left(y_{n}-p_{n}\right)^{2} \\
+ & \frac{1}{2} \sum_{n=1}^{N} \mathbf{k}\left(\left(p_{n}, q_{n}, x_{n}\right),\left(p_{n}, q_{n}, x_{n}\right)\right)\left(1-2 p_{n}\right)\left(y_{n}-p_{n}\right) \\
= & \frac{1}{2} \sum_{n=1}^{N} \sum_{i=1}^{N} \mathbf{k}\left(\left(p_{n}, q_{n}, x_{n}\right),\left(p_{i}, q_{i}, x_{i}\right)\right)\left(y_{n}-p_{n}\right)\left(y_{i}-p_{i}\right)
\end{aligned}
$$




$$
\begin{gathered}
-\frac{1}{2} \sum_{n=1}^{N} \mathbf{k}\left(\left(p_{n}, q_{n}, x_{n}\right),\left(p_{n}, q_{n}, x_{n}\right)\right) p_{n}\left(1-p_{n}\right) \\
=\frac{1}{2}\left\|\sum_{n=1}^{N}\left(y_{n}-p_{n}\right) \Phi\left(p_{n}, q_{n}, x_{n}\right)\right\|^{2}-\frac{1}{2} \sum_{n=1}^{N} p_{n}\left(1-p_{n}\right)\left\|\Phi\left(p_{n}, q_{n}, x_{n}\right)\right\|^{2},
\end{gathered}
$$

which immediately implies (13).

Theorem 3 allows us to prove the required results about separate calibration and resolution, decoupling Theorem 1s calibration-cum-resolution. The case of calibration will be trivial: we will just take the feature mapping $(p, q) \mapsto \operatorname{Exp}_{G}(p, q)$, where $G$ is the choice function. Resolution is covered by the following version of Theorem [1.e., Theorem 2 in [1]):

Theorem 4 The predictions $p_{n}$ output by the K29* algorithm with the object N91 kernel as parameter satisfy

$$
\left|\sum_{n=1}^{N}\left(y_{n}-p_{n}\right) f\left(x_{n}\right)\right| \leq\left(\frac{2}{\sqrt{3}}\right)^{K}\|f\|_{\mathrm{FS}} \sqrt{\sum_{n=1}^{N} p_{n}\left(1-p_{n}\right)} .
$$

for all $N$ and all Lipschitzian functions $f:[0,1]^{K} \rightarrow \mathbb{R}$.

The proof of this result is completely analogous to the proof of Theorem 2 in [11] (cf. the comments about the object N91 kernel in the previous subsection), and we do not reproduce it here. We will complement each prediction $p_{n} \in[0,1]$ to $\left(p_{n}, q_{n}\right) \in £$ choosing $q_{n} \in[0,1]$ arbitrarily.

To achieve both calibration and resolution, we will have to mix the feature mapping $\Phi_{0}(x, p, q):=\operatorname{Exp}_{G}(p, q)$ and the feature mapping $\Phi_{1}$ leading to the object N91 kernel. The following version of Corollary 2 in [10] gives a simple way to mix two feature mappings.

Corollary 5 Let $\Phi_{j}: £ \times \mathbf{X} \rightarrow H_{j}, j=0,1$, be admissible mappings from $£ \times \mathbf{X}$ to inner product spaces $H_{j}$. The lexicographic K29* algorithm with a suitable kernel parameter satisfies

$$
\begin{aligned}
&\left\|\sum_{n=1}^{N}\left(y_{n}-p_{n}\right) \Phi_{j}\left(p_{n}, q_{n}, x_{n}\right)\right\|^{2} \\
& \leq \sum_{n=1}^{N} p_{n}\left(1-p_{n}\right)\left(\left\|\Phi_{0}\left(p_{n}, q_{n}, x_{n}\right)\right\|^{2}+\left\|\Phi_{1}\left(p_{n}, q_{n}, x_{n}\right)\right\|^{2}\right)
\end{aligned}
$$

for all $N$ and for both $j=0$ and $j=1$.

Proof Define the direct sum $H$ of $H_{0}$ and $H_{1}$ as the Cartesian product $H_{0} \times H_{1}$ equipped with the inner product

$$
h \cdot h^{\prime}=\left(h_{0}, h_{1}\right) \cdot\left(h_{0}^{\prime}, h_{1}^{\prime}\right):=\sum_{j=0}^{1} h_{j} \cdot h_{j}^{\prime} .
$$


Now we can define $\Phi: £ \times \mathbf{X} \rightarrow H$ by

$$
\Phi(p, q, x):=\left(\Phi_{0}(p, q, x), \Phi_{1}(p, q, x)\right) ;
$$

the corresponding kernel is

$$
\begin{aligned}
\mathbf{k}\left((p, q, x),\left(p^{\prime}, q^{\prime}, x^{\prime}\right)\right) & :=\Phi(p, q, x) \cdot \Phi\left(p^{\prime}, q^{\prime}, x^{\prime}\right) \\
& =\sum_{j=0}^{1} \Phi_{j}(p, q, x) \cdot \Phi_{j}\left(p^{\prime}, q^{\prime}, x^{\prime}\right)=\sum_{j=0}^{1} \mathbf{k}_{j}\left((p, q, x),\left(p^{\prime}, q^{\prime}, x^{\prime}\right)\right),
\end{aligned}
$$

where $\mathbf{k}_{0}$ and $\mathbf{k}_{1}$ are the kernels corresponding to $\Phi_{0}$ and $\Phi_{1}$, respectively. It is clear that this kernel is admissible. Applying lexicographic K29* to it and using (13), we obtain

$$
\begin{aligned}
& \left\|\sum_{n=1}^{N}\left(y_{n}-p_{n}\right) \Phi_{j}\left(p_{n}, q_{n}, x_{n}\right)\right\|^{2} \\
& \leq\left\|\left(\sum_{n=1}^{N}\left(y_{n}-p_{n}\right) \Phi_{0}\left(p_{n}, q_{n}, x_{n}\right), \sum_{n=1}^{N}\left(y_{n}-p_{n}\right) \Phi_{1}\left(p_{n}, q_{n}, x_{n}\right)\right)\right\|^{2} \\
& =\left\|\sum_{n=1}^{N}\left(y_{n}-p_{n}\right) \Phi\left(p_{n}, q_{n}, x_{n}\right)\right\|^{2} \leq \sum_{n=1}^{N} p_{n}\left(1-p_{n}\right)\left\|\Phi\left(p_{n}, q_{n}, x_{n}\right)\right\|^{2} \\
& \quad=\sum_{n=1}^{N} p_{n}\left(1-p_{n}\right) \sum_{j=0}^{1}\left\|\Phi_{j}\left(p_{n}, q_{n}, x_{n}\right)\right\|^{2} .
\end{aligned}
$$

\section{Proof proper}

Let us take $\Phi_{0}(p, q, x):=\operatorname{Exp}_{G}(p, q)$ and $\Phi_{1}(p, q, x):=\left(f_{m}(x)\right)_{m \in M}$, the latter as in the proof of Theorem [4 (see [11, Appendix B). Remember that $\left\|\Phi_{1}(p, q, x)\right\|_{a} \leq 1, \forall p, q, x$. Merging $\Phi_{0}$ and $\Phi_{1}$ by Corollary [5] we obtain from the proof of Theorem 4 (namely, following the proof of Lemma 2 in [11; $c_{m}$ are the Fourier coefficients of $f$ w.r. to the system $\left.\left(f_{m}\right)\right)$ :

$$
\begin{aligned}
\left|\sum_{n=1}^{N}\left(y_{n}-p_{n}\right) f\left(x_{n}\right)\right|=\left|\sum_{m \in M} c_{m} \sum_{n=1}^{N}\left(y_{n}-p_{n}\right) f_{m}\left(x_{n}\right)\right| \\
=\left|\left(\sum_{n=1}^{N}\left(y_{n}-p_{n}\right) f_{m}\left(x_{n}\right)\right)_{m \in M} \cdot\left(\frac{c_{m}}{a_{m}}\right)_{m \in M}\right| \\
\leq\left\|\left(\sum_{n=1}^{N}\left(y_{n}-p_{n}\right) f_{m}\left(x_{n}\right)\right)_{m \in M}\right\|\left\|_{a}\right\|\left(\frac{c_{m}}{a_{m}}\right)_{m \in M} \|_{a} \\
=\left\|\sum_{n=1}^{N}\left(y_{n}-p_{n}\right) \Phi_{1}\left(p_{n}, q_{n}, x_{n}\right)\right\| \sqrt{\sum_{m \in M} \frac{c_{m}^{2}}{a_{m}}}
\end{aligned}
$$




$$
\leq \sqrt{\sum_{n=1}^{N} p_{n}\left(1-p_{n}\right)\left(\operatorname{Exp}_{G}^{2}\left(p_{n}, q_{n}\right)+1\right)}\left(\frac{2}{\sqrt{3}}\right)^{K}\|f\|_{\mathrm{FS}} .
$$

Finally, analogously to (3) and using (16), we obtain for the lexicographic K29* algorithm with the merged kernel as parameter:

$$
\begin{aligned}
& \sum_{n=1}^{N} \lambda\left(y_{n}, \gamma_{n}\right)=\sum_{n=1}^{N} \lambda\left(y_{n}, G\left(p_{n}, q_{n}\right)\right) \\
& =\sum_{n=1}^{N} \lambda\left(p_{n}, G\left(p_{n}, q_{n}\right)\right)+\sum_{n=1}^{N}\left(\lambda\left(y_{n}, G\left(p_{n}, q_{n}\right)\right)-\lambda\left(p_{n}, G\left(p_{n}, q_{n}\right)\right)\right) \\
& =\sum_{n=1}^{N} \lambda\left(p_{n}, G\left(p_{n}, q_{n}\right)\right)+\sum_{n=1}^{N}\left(y_{n}-p_{n}\right)\left(\lambda\left(1, G\left(p_{n}, q_{n}\right)\right)-\lambda\left(0, G\left(p_{n}, q_{n}\right)\right)\right) \\
& \leq \sum_{n=1}^{N} \lambda\left(p_{n}, G\left(p_{n}, q_{n}\right)\right)+\sqrt{\sum_{n=1}^{N} p_{n}\left(1-p_{n}\right)\left(\operatorname{Exp}_{G}^{2}\left(p_{n}, q_{n}\right)+1\right)} \\
& \leq \sum_{n=1}^{N} \lambda\left(p_{n}, D\left(x_{n}\right)\right)+N \Delta_{G}+\sqrt{\sum_{n=1}^{N} p_{n}\left(1-p_{n}\right)\left(\operatorname{Exp}_{G}^{2}\left(p_{n}, q_{n}\right)+1\right)} \\
& =\sum_{n=1}^{N} \lambda\left(y_{n}, D\left(x_{n}\right)\right)-\sum_{n=1}^{N}\left(\lambda\left(y_{n}, D\left(x_{n}\right)\right)-\lambda\left(p_{n}, D\left(x_{n}\right)\right)\right) \\
& +N \Delta_{G}+\sqrt{\sum_{n=1}^{N} p_{n}\left(1-p_{n}\right)\left(\operatorname{Exp}_{G}^{2}\left(p_{n}, q_{n}\right)+1\right)} \\
& =\sum_{n=1}^{N} \lambda\left(y_{n}, D\left(x_{n}\right)\right)-\sum_{n=1}^{N}\left(y_{n}-p_{n}\right)\left(\lambda\left(1, D\left(x_{n}\right)\right)-\lambda\left(0, D\left(x_{n}\right)\right)\right) \\
& +N \Delta_{G}+\sqrt{\sum_{n=1}^{N} p_{n}\left(1-p_{n}\right)\left(\operatorname{Exp}_{G}^{2}\left(p_{n}, q_{n}\right)+1\right)} \\
& \leq \sum_{n=1}^{N} \lambda\left(y_{n}, D\left(x_{n}\right)\right)+\left(\frac{2}{\sqrt{3}}\right)^{K}\left\|\operatorname{Exp}_{D}\right\|_{\mathrm{FS}} \sqrt{\sum_{n=1}^{N} p_{n}\left(1-p_{n}\right)\left(\operatorname{Exp}_{G}^{2}\left(p_{n}, q_{n}\right)+1\right)} \\
& +N \Delta_{G}+\sqrt{\sum_{n=1}^{N} p_{n}\left(1-p_{n}\right)\left(\operatorname{Exp}_{G}^{2}\left(p_{n}, q_{n}\right)+1\right)} \\
& =\sum_{n=1}^{N} \lambda\left(y_{n}, D\left(x_{n}\right)\right)+N \Delta_{G}
\end{aligned}
$$




$$
+\left(\left(\frac{2}{\sqrt{3}}\right)^{K}\left\|\operatorname{Exp}_{D}\right\|_{\mathrm{FS}}+1\right) \sqrt{\sum_{n=1}^{N} p_{n}\left(1-p_{n}\right)\left(\operatorname{Exp}_{G}^{2}\left(p_{n}, q_{n}\right)+1\right)} .
$$

\section{Appendix B: Details of the proof of Corollary 3}

In this appendix we will consider specific issues arising for unbounded games, concentrating on fixing the bug in the proof of Corollary 3

The stripped lexicographic square is the subset

$$
\varliminf^{\dagger}:=(0,1) \times[0,1]
$$

of $£$; the order and topology on $£^{\dagger}$ are inherited from $£$. The following analogue of Lemma 1 still holds.

Lemma 2 If a continuous function $f: £^{\dagger} \rightarrow \mathbb{R}$ takes both positive and negative values, it also takes the value 0 .

Proof See the proof of Lemma $f\left(f^{\dagger}\right)$ is still a connected set in $\mathbb{R}$.

A kernel $\mathbf{k}$ on $£^{\dagger} \times \mathbf{X}$ is admissible if the functions $\mathbf{k}\left((p, q, x),\left(p^{\prime}, q^{\prime}, x^{\prime}\right)\right)$ and $\mathbf{k}((p, q, x),(p, q, x))$ are continuous in $(p, q) \in £^{\dagger}$. The function (11) is then continuous in $(p, q) \in £^{\dagger}$, and for the kernel

$$
\begin{aligned}
\mathbf{k}\left((p, q, x),\left(p^{\prime}, q^{\prime}, x^{\prime}\right)\right) & \\
& =\operatorname{Exp}_{G}(p, q) \operatorname{Exp}_{G}\left(p^{\prime}, q^{\prime}\right)+\left(f_{m}(x)\right)_{m \in M} \cdot\left(f_{m}\left(x^{\prime}\right)\right)_{m \in M}
\end{aligned}
$$

used in the proof of Theorem 2 it equals

$$
\begin{aligned}
& S_{n}(p, q) \\
& =\sum_{i=1}^{n-1}\left(\operatorname{Exp}_{G}(p, q) \operatorname{Exp}_{G}\left(p_{i}, q_{i}\right)+\left(f_{m}\left(x_{n}\right)\right)_{m \in M} \cdot\left(f_{m}\left(x_{i}\right)\right)_{m \in M}\right)\left(y_{i}-p_{i}\right) \\
& \quad+\frac{1}{2}\left(\operatorname{Exp}_{G}^{2}(p, q)+\left\|\left(f_{m}\left(x_{n}\right)\right)_{m \in M}\right\|^{2}\right)(1-2 p) \\
& \quad=A \operatorname{Exp}_{G}(p, q)+B+\frac{1}{2} \operatorname{Exp}_{G}^{2}(p, q)(1-2 p)+C p
\end{aligned}
$$

where $A, B$, and $C$ do not depend on $(p, q)$. For the log loss game,

$$
\left|\operatorname{Exp}_{G}(p, q)\right|=\left|\ln \frac{1-p}{p}\right| \rightarrow \infty
$$

as $p \rightarrow 0$ or $p \rightarrow 1$, and so

$$
\lim _{\substack{(p, q) \rightarrow(1,0) \\(p, q) \in \in^{\dagger} \AA^{\dagger}}} S_{n}(p, q)=-\infty
$$


and

$$
\lim _{\substack{(p, q) \rightarrow(0,1) \\(p, q) \in \in^{\dagger}}} S_{n}(p, q)=\infty .
$$

The stripped lexicographic K29* algorithm (or, briefly, ${ }^{\dagger} \AA^{\dagger} \mathrm{K} 29^{*}$ ) is defined as the lexicographic K29* algorithm except that:

- its parameter is an admissible kernel $\mathbf{k}$ on $£^{\dagger} \times \mathbf{X}$;

- it outputs a root $(p, q) \in \mathscr{L}^{\dagger}$ of the equation $S_{n}(p, q)=0$ as $\left(p_{n}, q_{n}\right)$ and crashes if this equation does not have such roots (we will make sure this never happens).

Because of (19) and (20), the ${ }^{\dagger} \aleph^{\dagger} \mathrm{K} 29^{*}$ algorithm applied to the kernel (17) on $t^{\dagger} \times \mathbf{X}$ still ensures that (13) holds for the feature mapping $\left(\Phi_{0}, \Phi_{1}\right)$ used in the proof of Theorem [2 of course, this algorithm never outputs $\left(p_{n}, q_{n}\right)$ with $p_{n} \in\{0,1\}$. We can see that the proof of Theorem 2 still works. This completes the proof of Corollary 3

\section{Appendix C: Proof of Corollary 4}

Let us say that a straight line $(1-p) x+p y=c$ in the $(x, y)$-plane, where $p \in[0,1]$ and $c \in \mathbb{R}$, is southwest of the superdecision set $\Sigma$ if

$$
\forall(x, y) \in \Sigma:(1-p) x+p y \geq c .
$$

For each $p \in[0,1]$ let $c(p)$ be the largest $c$ (which obviously exists) such that the line $(1-p) x+p y=c$ is southwest of $\Sigma$. It is clear that, for $p \in(0,1)$, the line $(1-p) x+p y=c(p)$ intersects $\Sigma$ and the intersection, being compact and convex, has the form $[A(p), B(p)]$, where $A(p)$ and $B(p)$ are points (perhaps $A(p)=B(p))$ on the line. For concreteness, let $A(p)$ be northwest of $B(p)$ (i.e., if $A(p)=\left(A_{0}, A_{1}\right)$ and $B(p)=\left(B_{0}, B_{1}\right)$, we assume that $A_{0} \leq B_{0}$ and $\left.A_{1} \geq B_{1}\right)$. Now we can define the choice function $G$ as follows:

- if $0<p<1$ and $q \in[0,1], G(p, q)$ is defined to be any $\gamma \in \Gamma$ satisfying

$$
(\lambda(0, \gamma), \lambda(1, \gamma))=(1-q) A(p)+q B(p) ;
$$

the existence of such a $\gamma$ is obvious;

- if $p=0$ and $q \in[0,1], G(p, q)$ is defined to be any $\gamma \in \Gamma$ satisfying

$$
(\lambda(0, \gamma), \lambda(1, \gamma))=\left(C_{0}, f\left(C_{0}\right)\right) ;
$$

if $f\left(C_{0}\right)=\infty$, such a $\gamma$ does not exist and $G(p, q)$ is undefined;

- if $p=1$ and $q \in[0,1], G(p, q)$ is defined to be any $\gamma \in \Gamma$ such that

$$
(\lambda(0, \gamma), \lambda(1, \gamma))=\left(g\left(C_{1}\right), C_{1}\right) ;
$$

if $g\left(C_{1}\right)=\infty$, such a $\gamma$ does not exist and $G(p, q)$ is undefined. 
It is easy to see that the function $(\lambda(0, G(p, q)), \lambda(1, G(p, q)))$ is continuous in $(p, q) \in \operatorname{dom} G$ and, therefore, $\operatorname{Exp}_{G}(p, q)$ is continuous in $(p, q) \in \operatorname{dom} G$. We defined $G$ in such a way that it is a perfect choice function: $\Delta_{G}=0$.

Let us define the left-stripped lexicographic K29* algorithm (†£ 29 for brief) as the lexicographic K29* algorithm except that:

- its parameter is an admissible kernel $\mathbf{k}$ on $₫ £ \times \mathbf{X}$, where the left-stripped lexicographic square

$$
\dagger:=(0,1] \times[0,1]
$$

is equipped with the order and topology inherited from $£$;

- it outputs a root $(p, q) \in{ }^{\dagger} £$ of the equation $S_{n}(p, q)=0$ as $\left(p_{n}, q_{n}\right)$; if this equation does not have roots in $£$, we set $p_{n}:=1$ and set $q_{n} \in$ $[0,1]$ arbitrarily (we will make sure that this happens only when $S_{n}$ is everywhere positive).

In a similar way we define the right-stripped lexicographic K29* algorithm $\left(£^{\dagger} \mathrm{K} 29^{*}\right)$, which always outputs $\left(p_{n}, q_{n}\right) \in £^{\dagger}$, and the right-stripped lexicographic square $£^{\dagger}$; when $S_{n}(p, q)=0$ does not have roots $(p, q) \in £^{\dagger}$ we now set $p_{n}:=0$.

Let us first consider the case $\operatorname{dom} G=£$ (essentially, this is the case of a bounded loss function; the reservation "essentially" is needed since $\Gamma$ can contain "litter" - decisions dominated by other decisions in $\Gamma$ ). Since $G$ is continuous and $£$ is compact,

$$
\sup _{(p, q) \in £} p(1-p)\left(\operatorname{Exp}_{G}^{2}(p, q)+1\right)<\infty ;
$$

the application of (4) completes the proof in this case.

Let us now consider the case $\operatorname{dom} G=\dagger^{\dagger}$. First notice that, since $\left|\operatorname{Exp}_{G}(p, q)\right| \rightarrow 0$ both as $(p, q) \rightarrow(0,1)$ and as $(p, q) \rightarrow(1,0)$, 18) implies that the stripped lexicographic K29* algorithm never crashes. It remains to check that

$$
\limsup _{\substack{(p, q) \rightarrow(0,1) \\(p, q) \in^{\dagger} £^{\dagger}}} p \operatorname{Exp}_{G}^{2}(p, q)<\infty
$$

and

$$
\limsup _{\substack{(p, q) \rightarrow(1,0) \\(p, q) \in^{\dagger} £^{\dagger}}}(1-p) \operatorname{Exp}_{G}^{2}(p, q)<\infty .
$$

For example, let us demonstrate (22). Without loss of generality, we replace (10) with

$$
f_{-}^{\prime}(t)=O\left(t^{-2}\right), \quad g_{-}^{\prime}(t)=O\left(t^{-2}\right)
$$

(this can be done since $f_{-}^{\prime}(t) \leq f_{+}^{\prime}(t) \leq f_{-}^{\prime}(t+1)$ and $g_{-}^{\prime}(t) \leq g_{+}^{\prime}(t) \leq g_{-}^{\prime}(t+1)$ ). Consider the decision

$$
(X, Y):=(\lambda(0, G(p, q)), \lambda(1, G(p, q))) .
$$


Since $-\frac{1-p}{p}$ is a subgradient (see, e.g., 7], Section 23) of $f(x)$ at $X$, (23) implies that $1-p=O\left(X^{-2}\right)$, i.e., $(1-p) X^{2}=O(1)$. Since $\left|\operatorname{Exp}_{G}(p, q)\right|=X-Y \leq$ $X-C_{1}$ for $(p, q)<(1,0)$ sufficiently close to $(1,0)$, 22) indeed holds.

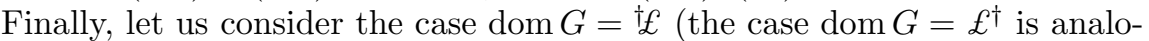
gous). In this case, $f\left(C_{0}\right)=\infty$ and $\operatorname{Exp}_{G}(p, q) \rightarrow \infty$ as $(p, q) \rightarrow(0,1)$. Since $S_{n}$ is continuous, the absence of roots of $S_{n}=0$ in $£$ means that $S_{n}$ is positive everywhere on ${ }^{\dagger}$, and so setting $p_{n}:=1$ in this case guarantees that ${ }^{\dagger} \mathrm{K} 29^{*}$ still ensures (13). It remains to notice that (21) still holds. 